\title{
Receiver operating characteristic curve analysis of the timed up and go test as a predictive tool for fall risk in persons with stroke: a retrospective study
}

\author{
Seung-yeop Lim ${ }^{a}$, Byung-jun Lee ${ }^{b}$, Wan-hee Lee ${ }^{b}$ \\ ${ }^{a}$ Education Team, Apsuninc, Seoul, Republic of Korea \\ ${ }^{\mathrm{b}}$ Department of Physical Therapy, College of Health Science and Social Welfare, Sahmyook University, Seoul, Republic of Korea
}

\begin{abstract}
Objective: Persons with chronic stroke fall more often than healthy elderly individuals. The Timed Up and Go test (TUG) is used as a fall prediction tool, but only provides a result for the total measurement time. This study aimed to determine the optimal cut-off values for each of the 6 components of the TUG.
\end{abstract}

Design: Retrospective study.

Methods: Thirty persons with chronic stroke participated in the study. TUG evaluation was performed using a wearable miniaturized inertial sensor. Sensitivity, specificity, and predictive values were calculated using the Receiver Operating Characteristic (ROC) curve analysis for the measured values in each section. Optimal values for fall risk classification were determined. Logistic regression analysis was used to investigate the risk of future falls based on TUG.

Results: The cut-off values of the 6 sections of the TUG were determined, as follows: sit-to-stand $>2.00$ seconds $(p<0.05)$, forward gait $>4.68$ seconds $(p<0.05)$, mid-turn $>3.82$ seconds $(p<0.05)$, return gait $>4.81$ seconds $(p<0.05)$, end-turn $>2.95$ seconds $(p<0.05)$, and stand-to-sit $>2.13$ seconds $(p<0.05)$. The risk of falling increased by 2.278 times when the mid-turn value was $>3.82$ seconds $(p<0.05)$.

Conclusions: The risk of falls increased by 2.28 times when the value of the mid-turn interval exceeded 3.82 seconds. Therefore, when interpreting TUG results, the predictive accuracy for falls will be higher when the measurement time for each section is analyzed, together with the total time for TUG.

Key Words: Accidental falls, Receiver operating characteristic curve, Walking

\section{Introduction}

A fall is an unintended change in posture, meaning that balance is lost and a part of the body touches the floor [1,2]. Falls are associated with a variety of negative consequences, including fractures, decreased physical functioning, fear of additional falls, and reduced quality of life [3,4]. Medical costs due to falls are rising year by year, and are expected to increase with aging [5]. Falls occur at a greater rate in stroke patients than in healthy elderly individuals $[1,6,7]$.
Stroke is a type of cerebrovascular disease caused by blood supply disorders or bleeding. Stroke survivors are more susceptible to falls than normal elderly individuals due to muscle paralysis, decreased balance ability, gait disturbance, and cognitive impairment $[1,6]$. Previous studies reported a fall rate of $11 \%$ to $30 \%$ in healthy elderly individuals, a rate of $25 \%$ in persons with subacute stroke, and a rate of $23 \%$ to $50 \%$ in persons with chronic stroke $[1,7]$. Risk of falls can be predicted using clinical testing [3]. The Berg Balance Scale, sit-to-stand test, Timed Up and Go Test

Received: 27 March, 2018 Revised: 21 April, 2018 Accepted: 26 April, 2018

Corresponding author: Wan-hee Lee (ORCID http://orcid.org/0000-0001-8030-4853)

Department of Physical Therapy, College of Health Science and Social Welfare, Sahmyook University, 815 Hwarang-ro, Nowon-gu, Seoul 01795, Republic of Korea

Tel: 82-2-3399-1633 Fax: 82-2-3399-1639 E-mail: whlee@syu.ac.kr

(c) This is an Open-Access article distributed under the terms of the Creative Commons Attribution Non-Commercial License (http://creativecommons.org/licenses/ by-nc/4.0) which permits unrestricted non-commercial use, distribution, and reproduction in any medium, provided the original work is properly cited.

Copyright $\odot 2018$ Korean Academy of Physical Therapy Rehabilitation Science 
(TUG), functional reach test, and maximal step length are used for fall prediction [8-10].

Among these, TUG is an effective and reliable verification tool for fall prediction and is widely used $[1,11]$. TUG measures the time required to stand up from a chair, walk 3 meters, and return to sit on the chair $[1,11,12]$. According to the Receiver Operating Characteristic (ROC) curve analysis for TUG fall prediction, the cut-off value (COV) was found to be 12.6 seconds in healthy elderly people and 21.42 seconds in persons with subacute stroke $[1,3,8]$. Although most studies only predict falls using the total measurement time of TUG, the motion sequence is complex: (1) stand up from a sitting position (in a chair), (2) forward gait for 3 meters, (3) rotate $180^{\circ}$ around a specific target, (4) return gait for 3 meters, (5) rotate $180^{\circ}$ in front of the chair, and (6) sit on the chair $[13,14]$. Therefore, if the TUG result is analyzed only for the total time, the analysis may be incorrect. In other words, if the total time is the same even though the time of each section is different, the risk of falls can be predicted incorrectly. Reinfelder et al. [13] reported the values for the 6 TUG sections in persons with Parkinson's disease [13]. However, no study has evaluated the risk of falls by measuring the 6 TUG sections in persons with chronic stroke. In addition, no study has analyzed the COVs using the ROC curve analysis for the 6 sections. Therefore, this study aimed to analyze the difference between faller and non-faller groups and to determine the optimum COV of the 6 TUG sections using ROC curves in persons with chronic stroke.

\section{Methods}

\section{Subjects and procedures}

This study was conducted on 30 chronic stroke survivors (20 males, 10 females) hospitalized in Saeall Rehabilitation Hospital. The inclusion criteria were as follows: (1) chronic stroke participants with hemiplegia (more than 6 months), (2) patients who could walk independently regardless of the use of assistive devices such as a cane, (3) patients with a Korean-language simplified psychiatric test (Mini-Mental State Examination-Korean version, MMSE-K) score $\geq 24$, and (4) patients who understood the study and agreed to participate. The exclusion criteria were as follows: (1) patients with a risk of falling when performing the study, (2) patients with unstable health status, (3) patients taking drugs that affect balance, (4) patients with unilateral neglect, or (5) patients with cognitive, visual, or sensory disturbances.
$[1,3]$.

This was a retrospective study in which falls were recorded based on the time since the onset of stroke [1]. The general characteristics of the subjects (sex, age, weight, height, and stroke duration) were taken from medical record review with approval of hospital personnel. Falls were defined as unexpected postural changes with unintentional loss of balance in which part of the body reached the floor [2]. A previous study, the "faller group" had experienced 2 or more falls since stroke onset while the "non-faller group" had experienced only 1 fall or no falls since stroke onset [15]. The purpose of this study was explained in detail and the subjects were advised they could withdraw at any time. The study protocol was approved by the Institutional Review Board of Sahmyook University in Seoul (IRB No. 2-1040781-AB-N01-2018005HR). Participants provided signed consent after receiving verbal and written information about the study.

\section{Measurement method and evaluation tool}

The TUG was performed to predict falls in those with chronic stroke. Subjects were observed and timed as they stood up from a chair, walked 3 meters, turned around, walked back to the chair, and sat down [3]. The inter-rater reliability of this test is $r=0.99$, and the intra-rater reliability is $\mathrm{r}=0.98$ [12]. The measurements were performed 3 times, and the average value was used.

The TUG components were measured using a single wearable miniaturized inertial sensor (G-Sensor; BTS Bioengineering S.p.A., Milano, Italy), as in previous studies [16]. This device is composed of a 16-bit/axis triaxial accelerometer with multiple sensitivities $( \pm 2, \pm 4, \pm 8$, and $16 \mathrm{~g}$ ), a 13-bit triaxial magnetometer $( \pm 1,200 \mu \mathrm{T})$, and a 16-bit/axis triaxial gyroscope with multiple sensitivities $( \pm 250, \pm 500$, $\pm 1,000$, and $\pm 2,000^{\circ} / \mathrm{s}$ ) [16]. The validity of the inertial sensor was reported by Bugane and colleagues [17]. This device was attached at the L4-5 level inter-vertebral space using a semi-elastic belt and collected the characteristics of acceleration and angle changes of paces along 3 orthogonal axes $[14,18,19]$. Data were collected at a sampling rate of $100 \mathrm{~Hz}$ and analyzed using dedicated software (G-Studio; BTS Bioengineering S.p.A.) (Figure 1).

\section{Statistical analysis}

Data were analyzed using IBM SPSS Statistics ver. 18.0 (IBM Co., Armonk, NY, USA). The chi-square test and independent $t$-test were used to compare the general characteristics and functional performance of faller and non-faller 
A

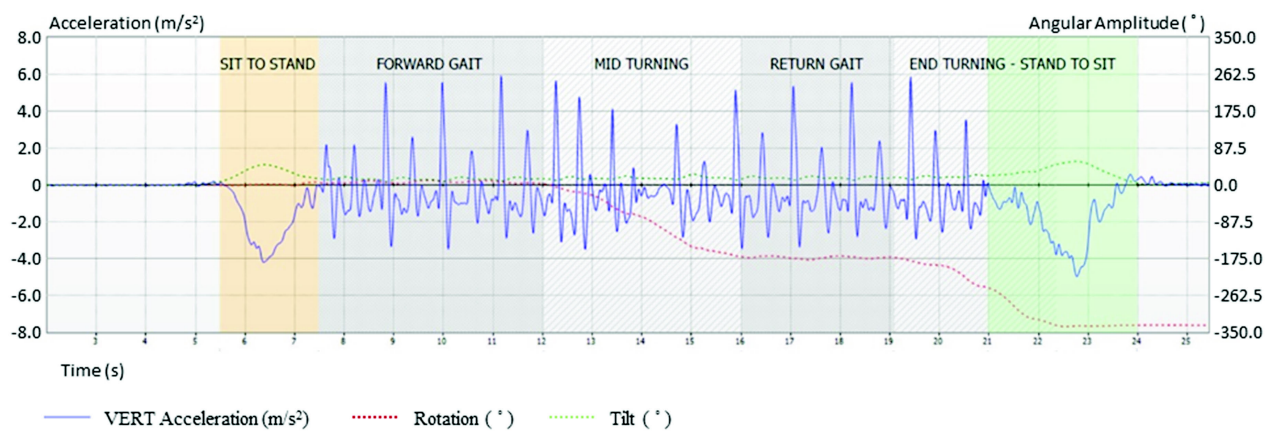

B

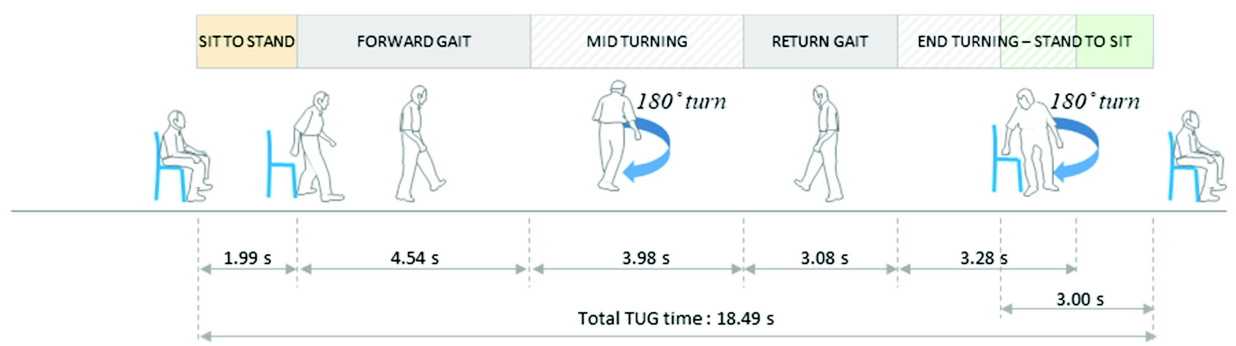

Figure 1. Timed Up and Go test (TUG) raw data measured using a single wireless inertial sensor. (A) Acceleration and angular amplitude graph. (B) TUG results.

groups. The effect size was computed using Cohen's d, a standardized mean (mean of non-faller group - mean of faller group/standard deviation of total group) of the non-faller group versus the faller group. Cohen's $d$ of $>0.8$ indicated that the effect size was large, $0.5<\mathrm{d} \leq 0.8$ was moderate, and $0.2<\mathrm{d} \leq 0.5$ was small [20].

$$
\text { Cohen's d }=\frac{\text { Mean of non-faller group }- \text { Mean of faller group }}{\text { Standard deviation of total group }}
$$

To determine the ROC curve, COV, positive predictive value, and negative predictive value for each of the 6 TUG sections, the MedCalc 14.8.1 Statistical Program (MedCalc Software, Ostend, Belgium) was used. For the area under the curve (AUC) of the ROC curve, $0.5<$ AUC $\leq 0.7$ was less accurate, $0.7<\mathrm{AUC} \leq 0.9$ was moderately accurate, $0.9<\mathrm{AUC}$ $<1.0$ was very accurate, and $\mathrm{AUC}=1$ was perfectly accurate [21]. The COV was used to calculate the odds ratio using logistic regression to estimate the degree of risk of falls. Statistical significance was set at 0.05 .

\section{Results}

\section{General characteristics and TUG measures of the faller and non-faller groups}

There was no significant difference in sex, age, body weight, height, and duration since the stroke incident between the faller and non-faller groups (Table 1). However, the faller group significantly took a longer time to complete both the total TUG (tTUG) and the 6 sections than the non-faller group $(p<0.05$; Tables 1,2$)$.

\section{COVs of fall predictors}

As a result of the ROC curve analysis, the AUC of all measured TUG values was 0.78 to 0.86 , showing moderate accuracy. The tTUG COV was 20.45 seconds (sensitivity $[\mathrm{SEN}]=92.31 \%$, specificity $[\mathrm{SPE}]=70.59 \%$ ), the sit-to-stand COV was 2.00 seconds ( $\mathrm{SEN}=76.92 \%, \mathrm{SPE}=82.35 \%)$, the forward gait $\mathrm{COV}$ was 4.68 seconds $(\mathrm{SEN}=92.31 \%$, $\mathrm{SPE}=$ $64.71 \%$ ), the mid-turn $\mathrm{COV}$ was 3.82 seconds ( $\mathrm{SEN}=$ $92.31 \%$, $\mathrm{SPE}=76.47 \%$ ), the return gait $\mathrm{COV}$ was $4.81 \mathrm{sec}-$ onds $(\mathrm{SEN}=84.62 \%, \mathrm{SPE}=70.59 \%$ ), the end-turn $\mathrm{COV}$ was 
Table 1. General characteristics of fallers and non-fallers

$(\mathrm{N}=30)$

\begin{tabular}{|c|c|c|c|c|c|c|}
\hline Characteristic & All & Faller $(n=13)$ & Non-faller $(n=17)$ & $\chi^{2 / t}$ & $p$-value & Cohen's d \\
\hline Sex (male/female) ${ }^{\mathrm{a}}$ & $20 / 10$ & $8 / 5$ & $12 / 5$ & 0.271 & 0.602 & - \\
\hline Age (y) & $58.53(11.62)$ & $61.69(16.08)$ & $56.12(6.04)$ & -1.188 & 0.254 & 0.479 \\
\hline Weight (kg) & $66.30(12.14)$ & $66.31(13.08)$ & $66.29(11.79)$ & -0.003 & 0.998 & $<0.001$ \\
\hline Height (m) & $1.66(0.11)$ & $1.64(0.13)$ & $1.68(0.08)$ & 0.957 & 0.347 & 0.364 \\
\hline Duration (mo) & $20.57(10.61)$ & $24.77(10.14)$ & $17.35(10.08)$ & -1.992 & 0.056 & 0.699 \\
\hline
\end{tabular}

Values are presented as number only or mean (SD).

${ }^{a}$ Chi-square.

Table 2. The timed up and go test results of fallers and non-fallers

$(\mathrm{N}=30)$

\begin{tabular}{lcccccc}
\hline Characteristic & All & Faller $(\mathrm{n}=13)$ & Non-faller (n=17) & $\mathrm{t}$ & $p$-value & Cohen's d \\
\hline tTUG (s) & $24.95(10.44)$ & $32.20(9.08)$ & $19.40(7.77)$ & -4.155 & $<0.001^{* *}$ & 1.23 \\
Sit-to-stand (s) & $2.06(0.54)$ & $2.37(0.64)$ & $1.83(0.29)$ & -2.825 & $0.012^{*}$ & 1.00 \\
Forward gait (s) & $6.10(2.95)$ & $8.06(2.65)$ & $4.60(2.23)$ & -3.889 & $0.001^{* *}$ & 1.17 \\
Mid-turn (s) & $4.91(2.37)$ & $6.62(2.29)$ & $3.60(1.42)$ & -4.444 & $<0.001^{* *}$ & 1.27 \\
Return gait (s) & $5.88(3.26)$ & $7.81(2.91)$ & $4.40(2.76)$ & -3.278 & $0.003^{* *}$ & 1.05 \\
End-turn (s) & $3.73(1.90)$ & $4.98(2.00)$ & $2.78(1.14)$ & -3.548 & $0.002^{* *}$ & 1.16 \\
Stand-to-sit (s) & $2.65(1.04)$ & $3.23(1.17)$ & $2.20(0.65)$ & -2.855 & $0.011^{*}$ & 0.99 \\
\hline
\end{tabular}

Values are presented as mean (SD). tTUG: total Timed Up and Go test. ${ }^{*} p<0.05, \stackrel{*}{* *} p<0.01$.

Table 3. Cut-off value (COV) of timed up and go test $(\mathrm{N}=30)$

\begin{tabular}{llllllllll}
\hline \multicolumn{1}{c}{ Characteristic } & COV & AUC $(95 \%$ CI) & SEN & SPE & $p$-value & PPV & NPV & + LR & - LR \\
\hline tTUG (s) & $>20.45$ & $0.86(0.68-0.96)$ & 92.31 & 70.59 & $0.001^{* *}$ & 70.6 & 92.3 & 3.14 & 0.11 \\
Sit-to-stand (s) & $>2.00$ & $0.78(0.60-0.91)$ & 76.92 & 82.35 & $0.004^{* *}$ & 76.9 & 82.4 & 4.36 & 0.28 \\
Forward gait (s) & $>4.68$ & $0.84(0.66-0.95)$ & 92.31 & 64.71 & $0.001^{* *}$ & 66.7 & 91.7 & 2.62 & 0.12 \\
Mid-turn (s) & $>3.82$ & $0.86(0.69-0.96)$ & 92.31 & 76.47 & $0.001^{* *}$ & 75.0 & 92.9 & 3.92 & 0.10 \\
Return gait (s) & $>4.81$ & $0.80(0.61-0.92)$ & 84.62 & 70.59 & $0.001^{* *}$ & 68.7 & 85.7 & 2.88 & 0.22 \\
End-turn (s) & $>2.95$ & $0.84(0.66-0.95)$ & 92.31 & 76.47 & $0.001^{* *}$ & 75.0 & 92.9 & 3.92 & 0.10 \\
Stand-to-sit (s) & $>2.13$ & $0.78(0.59-0.91)$ & 84.62 & 64.71 & $0.001^{* *}$ & 64.7 & 84.6 & 2.40 & 0.24 \\
\hline
\end{tabular}

AUC: area under the curve, CI: confidence interval, SEN: sensitivity, SPE: specificity, PPV: positive predictive value, NPV: negative predictive value, +LR: positive likelihood ratio, - LR: negative likelihood ratio, tTUG: total Timed Up and Go test.

${ }^{* *} p<0.01$.

2.95 seconds $(\mathrm{SEN}=92.31 \%, \mathrm{SPE}=76.47 \%)$, and the standto-sit $\mathrm{COV}$ was 2.13 seconds $(\mathrm{SEN}=84.62 \%$, $\mathrm{SPE}=64.71 \%)$ (Table 3, Figure 2).

\section{Bivariate logistic regression analysis of fall prediction} variables using the ROC curve

The results of bivariate logistic regression analysis for prediction of the risk of falling using the COV of each measurement variable showed that the mid-turn value was a significant predictor. Participants with chronic stroke with a mid-turn of $>3.82$ seconds were 2.278 times more likely to experience a fall compared with participants who did not ex- ceed 3.82 seconds (Table 4$)$.

\section{Discussion}

This study investigated the difference between faller and non-faller in persons with chronic stroke, and analyzed the optimal COV for each of the $6 \mathrm{COV}$ sections. ROC curve analysis showed that the COV of tTUG was 20.45 seconds and the AUC was 0.86. The COV for each of the $6 \mathrm{COV}$ sections was as follows: sit-to-stand, 2.00 seconds; forward gait, 4.68 seconds; mid-turn, 3.82 seconds; return gait, 4.81 seconds; end-turn, 2.95 seconds; and stand-to-sit, 2.13 

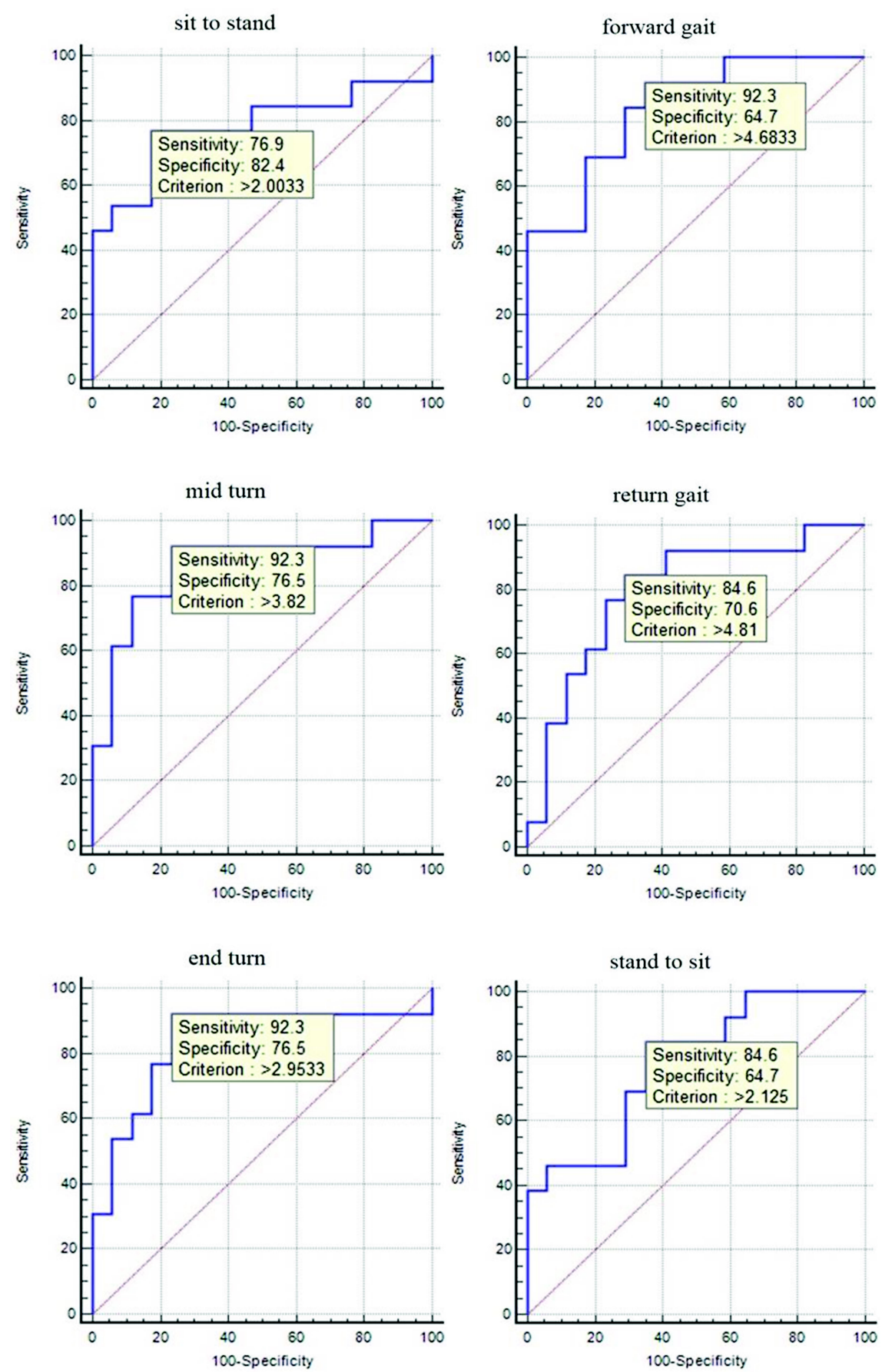

Figure 2. Receiver operating characteristic curve of Timed Up and Go test.

seconds. The AUC for each of the 6 sections was $\geq 0.78$, showing moderate accuracy. In addition, the risk of falling increased 2.28 times when the mid turn exceeded 3.82 seconds. This study showed that the COV for each section and the mid-turn value could provide more specific fall prediction, making a positive contribution to fall prevention activities.
Recently, a study has analyzed the TUG sections. Reinfelder et al. [13] proposed a method of subdividing and analyzing the TUG components for persons with Parkinson's disease. Two inertial sensors and a high-speed camera were used for this purpose. The inertial sensors were attached to each side of the shoe, and a segmentation studies was performed using a high-speed camera. According to the 
Table 4. Logistic regression analysis for predicting risk of falls

$(\mathrm{N}=30)$

\begin{tabular}{lrrcl}
\hline \multicolumn{1}{c}{ Variable } & B & $\begin{array}{c}\text { Standard } \\
\text { error }\end{array}$ & $\begin{array}{c}\text { Odds ratio } \\
(95 \% \mathrm{CI})\end{array}$ & $p$-value \\
\hline Sit-to-stand (s) & 0.99 & 1.75 & $2.69(0.09-83.30)$ & 0.573 \\
Forward gait (s) & 0.24 & 0.85 & $1.28(0.24-6.77)$ & 0.775 \\
Mid-turn (s) & 0.82 & 0.29 & $2.278(1.30-4.00)$ & $0.004^{* *}$ \\
Return gait (s) & -0.31 & 0.57 & $0.735(0.24-2.23)$ & 0.586 \\
End-turn (s) & 0.04 & 0.68 & $1.038(0.28-3.90)$ & 0.956 \\
Stand-to-sit (s) & -0.62 & 1.18 & $0.536(0.05-5.38)$ & 0.596 \\
\hline
\end{tabular}

CI: confidence interval. ${ }^{* *} p<0.01$.

results of the study, it was possible to distinguish between 'sit to walk', 'walking', 'first turn', 'second turn', and 'turn to sit', but did not distinguish between 'sit-to-stand' and 'stand to walk'. This may possibly be due to the sensitivity limit of the inertial sensor used in this study. The sensitivity of the accelerometer used by Reinfelder et al. [13] was $\pm 6 \mathrm{~g}$ and the sensitivity of the angular velocity was $\pm 1,000 \% \mathrm{~s}$. However, with the recent development of technology, the sensitivity of the acceleration sensor can be up to $\pm 16 \mathrm{~g}$, and the angular velocity can be measured up to $\pm 2,000 \%$ s. If further studies can be conducted using high-tech inertial sensors, the TUG COV of each item may be better distinguished in persons with Parkinson's disease.

Previous studies have calculated the COVs through ROC analysis of the TUG. According to Kojima et al. [3], the COV was 12.6 seconds and the AUC was 0.58 [3]. Killough [22] reported a COV of 12.2 seconds and an AUC of 0.64. Sai et al. [23] and Viccaro et al. [24] reported an AUC of $\geq$ 0.7. The COV and AUC values in previous studies were smaller than in this study. This is thought to be due to differences in the physical characteristics of the subjects participating in the study. In previous studies, the subjects were $>65$ years old and healthy. However, since this study was conducted on persons with chronic stroke with a stroke onset of more than 6 months, the COVs were somewhat higher.

To predict the risk of falls using the COV, the odds ratio was calculated using logistic regression analysis. The risk of falling was significantly increased at 2.28 times when the mid-turn value exceeded 3.82 seconds. The TUG has a mid-turn and end-turn [14], however, no significant results were obtained for the end-turn. This is thought to be due to the characteristics of the section where the turn occurs. Mid-turn occurs between forward gait and return gait. In other words, the mid-turn is where the direction change oc- curs due to continuous forward walking. Strike and Taylor [25] reported that direction change reduces the walking speed compared to that in straight gait and produces an asymmetric gait pattern. For those affected by stroke, redirection is associated with more steps and longer durations compared to healthy adults, and this finding was supported in this study [26,27]. The inertial sensor used can measure the change in acceleration and angle (Figure 1). Vertical acceleration (VA $\left[\mathrm{m} / \mathrm{s}^{2}\right]$ ) represents the change in the vertical speed of the pelvis during gait [28]. This value can be interpreted as symmetrical walking if it is evenly distributed around zero. However, in the case of participants with chronic stroke, asymmetric gait appears in the straight gait sections (forward/return gait). Moreover, during the mid-turn section, it can be seen that the rotation value increases in the negative direction, and the VA value decreases and then increases again (Figure 1). It is thought that this result is related to the decrease in walking speed due to the instability of the change in direction during the turn. Therefore, the mid-turn value can be considered as a high-risk section for falls.

The end-turn occurs between return gait and stand-to-sit. That is, the end-turn occurs simultaneously with sitting on a chair, which indicates that the raw TUG data shows that the end-turn section and the stand-to-sit section overlap (Figure 1). This means that the stand-to-sit operation will start during the end-turn. This section no longer shows forward gait. The pivot motion in front of the chair and forward bending of the trunk to sit on the chair occurs in this section. When moving to sit on the chair, the end-turn is terminated, the trunk extends back, and the stand-to-sit motion is terminated. That is, the end-turn is simultaneous with the stand-to-sit and becomes a more stable state. Because of this feature, the risk of falls in the end-turn is not thought to be significant.

A limitation of this study was that the history of falls after stroke was determined according to the patient's subjective response, which can be biased. For the TUG evaluation, the subjects who were able to walk independently were selected. However, the use of assistive devices could cause errors. In addition, only 30 subjects were included, making generalization to all chronic stroke survivors difficult. Therefore, future longitudinal studies to examine the COV of falls are needed.

This study showed the predictive value of the COVs for falls in each of the 6 TUG sections in those affected by chronic stroke. The risk for falls was increased by 2.28 times 
when the value of the mid-turn interval exceeded 3.82 seconds. Therefore, when interpreting the TUG results, the predictive accuracy for falls will be higher when the measurement time for each section is analyzed, together with the total TUG score.

\section{Conflict of Interest}

The authors declared no potential conflicts of interest with respect to the authorship and/or publication of this article.

\section{References}

1. Jung SH, Park DS, Kang TK, An SH. The discriminant analysis and predictive validity for predictor of falls using STS, TUG, FRT, MSL, BBS in patients with chronic stroke. J Spec Educ Rehabil Sci 2017;56:327-45.

2. Lamb SE, Jørstad-Stein EC, Hauer K, Becker C; Prevention of Falls Network Europe and Outcomes Consensus Group. Development of a common outcome data set for fall injury prevention trials: the Prevention of Falls Network Europe consensus. J Am Geriatr Soc 2005;53:1618-22.

3. Kojima G, Masud T, Kendrick D, Morris R, Gawler S, Treml J, et al. Does the timed up and go test predict future falls among British community-dwelling older people? Prospective cohort study nested within a randomised controlled trial. BMC Geriatr 2015;15:38.

4. Kumar A, Carpenter H, Morris R, Iliffe S, Kendrick D. Which factors are associated with fear of falling in community-dwelling older people? Age Ageing 2014;43:76-84.

5. Davis JC, Robertson MC, Ashe MC, Liu-Ambrose T, Khan KM, Marra CA. International comparison of cost of falls in older adults living in the community: a systematic review. Osteoporos Int 2010;21:1295-306.

6. Mackintosh SF, Hill KD, Dodd KJ, Goldie PA, Culham EG. Balance score and a history of falls in hospital predict recurrent falls in the 6 months following stroke rehabilitation. Arch Phys Med Rehabil 2006;87:1583-9.

7. Hyndman D, Ashburn A, Stack E. Fall events among people with stroke living in the community: circumstances of falls and characteristics of fallers. Arch Phys Med Rehabil 2002;83:165-70.

8. An SH, Song SH, Lee GC. A study for predicting the risk of falls in sub-acute stroke survivors. J Basic Sci 2015;32:47-57.

9. Park CS. The validity of the modified postural assessment scale for stroke and Berg Balance Scale in predicting fall of subacute stroke patients. J Spec Educ Rehabil Sci 2014;53:1-18.

10. Park CS, Kim WG, An SH. The study on predicting of fall incidence using the falls-related efficacy scale in people with chronic stroke. J Spec Educ Rehabil Sci 2013;52:241-58.

11. Gates S, Smith LA, Fisher JD, Lamb SE. Systematic review of accuracy of screening instruments for predicting fall risk among independently living older adults. J Rehabil Res Dev 2008;45:1105-16.
12. Podsiadlo D, Richardson S. The timed "Up \& Go": a test of basic functional mobility for frail elderly persons. J Am Geriatr Soc 1991;39:142-8

13. Reinfelder S, Hauer R, Barth J, Klucken J, Eskofier BM. Timed Up-and-Go phase segmentation in Parkinson's disease patients using unobtrusive inertial sensors. Conf Proc IEEE Eng Med Biol Soc 2015;2015:5171-4.

14. Lee G, Cho CH, Lim KJ, Lee JH, Yoon GR, Woo YK. Effect of direction to be used for the Timed Up and Go Test on walking time in stroke patients. Phy Ther Korea 2016;23:11-9.

15. Thomas JI, Lane JV. A pilot study to explore the predictive validity of 4 measures of falls risk in frail elderly patients. Arch Phys Med Rehabil 2005;86:1636-40.

16. Lameira de Melo GE, Franco de Moura RC, Lopes JBP, Junior PRF, Lazzari RD, Carvalho Duarte NA, et al. Effects of virtual reality on parkinsonian gait: blind controlled randomized clinical trial protocol. Man Ther Posturology Rehabil J 2017;15:524.

17. Bugané F, Benedetti MG, Casadio G, Attala S, Biagi F, Manca M, et al. Estimation of spatial-temporal gait parameters in level walking based on a single accelerometer: validation on normal subjects by standard gait analysis. Comput Methods Programs Biomed 2012;108:129-37.

18. Lorefice L, Coghe G, Fenu G, Porta M, Pilloni G, Frau J, et al. 'Timed up and go' and brain atrophy: a preliminary MRI study to assess functional mobility performance in multiple sclerosis. $\mathrm{J}$ Neurol 2017;264:2201-4.

19. Pau M, Porta M, Coghe G, Corona F, Pilloni G, Lorefice L, et al. Are static and functional balance abilities related in individuals with Multiple Sclerosis? Mult Scler Relat Disord 2017;15:1-6.

20. Cohen J. Statistical power analysis. Curr Dir Psychol Sci 1992;1:98-101.

21. Greiner M, Pfeiffer D, Smith RD. Principles and practical application of the receiver-operating characteristic analysis for diagnostic tests. Prev Vet Med 2000;45:23-41.

22. Killough J. Validation of the timed up and go test to predict falls. J Geriatr Phys Ther 2006;29:128-9.

23. Sai AJ, Gallagher JC, Smith LM, Logsdon S. Fall predictors in the community dwelling elderly: a cross sectional and prospective cohort study. J Musculoskelet Neuronal Interact 2010;10:142-50.

24. Viccaro LJ, Perera S, Studenski SA. Is timed up and go better than gait speed in predicting health, function, and falls in older adults? J Am Geriatr Soc 2011;59:887-92.

25. Strike SC, Taylor MJ. The temporal-spatial and ground reaction impulses of turning gait: is turning symmetrical? Gait Posture 2009;29:597-602.

26. Lamontagne A, Fung J. Gaze and postural reorientation in the control of locomotor steering after stroke. Neurorehabil Neural Repair 2009;23:256-66.

27. Mackintosh SF, Hill K, Dodd KJ, Goldie P, Culham E. Falls and injury prevention should be part of every stroke rehabilitation plan. Clin Rehabil 2005;19:441-51.

28. Zarzeczny R, Nawrat-Szołtysik A, Polak A, Maliszewski J, Kiełtyka A, Matyja B, et al. Aging effect on the instrumented Timed-Up-and-Go test variables in nursing home women aged 80-93 years. Biogerontology 2017;18:651-63. 